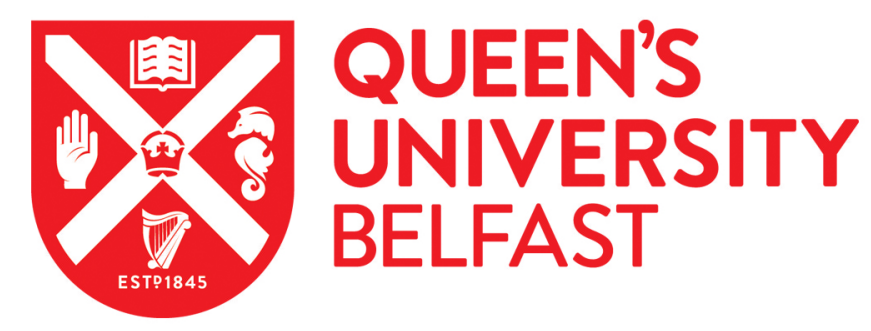

\title{
USP17 is required for peripheral trafficking of lysosomes
}

Lin, J., McCann, A., Sereesongsaeng, N., Burden, J., Alsa'd, A. A. M., Burden, R., Micu, I., Williams, R., Van Schaeybroeck, S., Evergren, E., Mullan, P., Scott, C., \& Burrows, J. (2022). USP17 is required for peripheral trafficking of lysosomes. EMBO Reports. https://doi.org/10.15252/embr.202051932

\section{Published in: \\ EMBO Reports}

Document Version:

Publisher's PDF, also known as Version of record

Queen's University Belfast - Research Portal:

Link to publication record in Queen's University Belfast Research Portal

\section{Publisher rights}

Copyright 2022 the authors.

This is an open access article published under a Creative Commons Attribution License (https://creativecommons.org/licenses/by/4.0/), which permits unrestricted use, distribution and reproduction in any medium, provided the author and source are cited.

\section{General rights}

Copyright for the publications made accessible via the Queen's University Belfast Research Portal is retained by the author(s) and / or other copyright owners and it is a condition of accessing these publications that users recognise and abide by the legal requirements associated with these rights.

Take down policy

The Research Portal is Queen's institutional repository that provides access to Queen's research output. Every effort has been made to ensure that content in the Research Portal does not infringe any person's rights, or applicable UK laws. If you discover content in the Research Portal that you believe breaches copyright or violates any law, please contact openaccess@qub.ac.uk. 


\title{
USP17 is required for peripheral trafficking of
} lysosomes

\author{
Jia Lin ${ }^{1}$, Aidan P McCann¹, Naphannop Sereesongsaeng ${ }^{1}$, Jonathan M Burden ${ }^{1}$, Alhareth A Alsa'd ${ }^{1}$, \\ Roberta E Burden ${ }^{1}$ (D), Ileana Micu² (D), Richard Williams ${ }^{3}$, Sandra Van Schaeybroeck ${ }^{3}$, Emma Evergren $^{3}$, \\ Paul Mullan ${ }^{3}$, Jeremy C Simpson ${ }^{4}$, Christopher J Scott ${ }^{3}$ \& James F Burrows ${ }^{1, *}$ (D)
}

\begin{abstract}
Expression of the deubiquitinase USP17 is induced by multiple stimuli, including cytokines (IL-4/6), chemokines (IL-8, SDF1), and growth factors (EGF), and several studies indicate it is required for cell proliferation and migration. However, the mechanisms via which USP17 impacts upon these cellular functions are unclear. Here, we demonstrate that USP17 depletion prevents peripheral lysosome positioning, as well as trafficking of lysosomes to the cell periphery in response to EGF stimulation. Overexpression of USP17 also increases secretion of the lysosomal protease cathepsin D. In addition, USP17 depletion impairs plasma membrane repair in cells treated with the pore-forming toxin streptolysin 0 , further indicating that USP17 is required for lysosome trafficking to the plasma membrane. Finally, we demonstrate that USP17 can deubiquitinate p62, and we propose that USP17 can facilitate peripheral lysosome trafficking by opposing the E3 ligase RNF26 to untether lysosomes from the ER and facilitate lysosome peripheral trafficking, lysosome protease secretion, and plasma membrane repair.
\end{abstract}

Keywords EGF; exocytosis; lysosome; USP17

Subject Categories Membranes \& Trafficking; Organelles; Post-translational Modifications \& Proteolysis

DOI 10.15252/embr.202051932 | Received 20 October 2020 | Revised 14

December 2021 | Accepted 23 December 2021

EMBO Reports (2022) e51932

\section{Introduction}

Lysosomes are no longer seen as just static "waste bags" which degrade unwanted proteins, or organelles, brought to them via endocytosis or autophagosomes. Instead, they are recognized as dynamic organelles which traffic throughout the cell and participate in important cellular processes such as nutrient sensing, autophagy, secretion, and plasma membrane repair (Samie \& Xu, 2014). Indeed, lysosomal-related functions such as secretion and plasma membrane repair require the lysosomes to relocate from the "perinuclear cloud" to the plasma membrane. These lysosomes can then fuse with the plasma membrane to release their contents into the extracellular space (Samie \& Xu, 2014).

The importance of lysosomes is further emphasized by accumulating evidence that lysosome dysfunction is associated with pathologies including neurodegenerative (Alzheimer's and Parkinson's disease) and autoimmune (rheumatoid arthritis and lupus) diseases, as well as cancer (Bonam et al, 2019), and upregulation of lysosomal proteins is a conserved feature of aging (Cellerino \& Ori, 2017). As a result, it is important that we understand how lysosome positioning is regulated, and what consequences this has on lysosome function.

The DUB/ubiquitin-specific protease 17 (USP17) family of deubiquitinases was originally identified in mice (DUB-1, DUB-1A, and DUB-2) (Zhu et al, 1996, 1997), and the human homolog, USP17/DUB-3/Dub3 (subsequently referred to as USP17), is induced in response to cytokine, chemokine, and epidermal growth factor (EGF) stimulation (Burrows et al, 2004; de la Vega et al, 2011; Jaworski et al, 2014). USP17 expression is required for proper G1 to S cell cycle progression (McFarlane et al, 2010), chemokine-driven (IL-8, SDF1) cell motility (de la Vega et al, 2011), and EGF receptor (EGFR) clathrin-mediated endocytosis (Jaworski et al, 2014). In addition, we and others have shown that USP17 is overexpressed in a range of tumors when compared to normal tissue (non-small cell lung cancer (NSCLC), ovarian, breast, colon, esophagus, cervical, and osteosarcoma) (McFarlane et al, 2010, 2013; Pereg et al, 2010; Zhou et al, 2015; Zhang et al, 2016; Song et al, 2017).

In this study, we show that USP17 is required for peripheral lysosome trafficking, as well as EGF triggered lysosome exocytosis. In addition, we demonstrate that, in the absence of USP17, the repair of plasma membrane damage is dramatically impeded, again indicating USP17 promotes peripheral lysosome trafficking, thus facilitating plasma membrane repair. Finally, we show that USP17 counteracts the impact of the E3 ligase RNF26 on lysosome trafficking by deubiquitinating its substrate p62, indicating it triggers

\footnotetext{
1 School of Pharmacy, Queen's University Belfast, Belfast, UK

2 Advanced Imaging Core Technology Unit, Faculty of Medicine, Health and Life Sciences, Queen's University Belfast, Belfast, UK

3 Patrick G Johnston Centre for Cancer Research, School of Medicine, Dentistry and Biomedical Sciences, Queen's University Belfast, Belfast, UK

4 School of Biology and Environmental Science, UCD Conway Institute of Biomolecular and Biomedical Research, University College Dublin, Dublin 4, Ireland

*Corresponding author. Tel: +44 2890972703; E-mail: j.burrows@qub.ac.uk
} 
peripheral lysosome trafficking by untethering them from the endoplasmic reticulum (ER).

\section{Results and Discussion}

\section{USP17 is required for peripheral trafficking of lysosomes}

A link between USP17 and the lysosome was previously suggested in two studies indicating USP17 deubiquitinates legumain to regulate its stability (Lin et al, 2014; Chen et al, 2019). However, we were intrigued how USP17, which we have previously shown to display a perinuclear distribution in HeLa cells (McFarlane et al, 2010), and to colocalize with the ER marker calnexin (Burrows et al, 2009), could directly impact a lysosomal protease and decided to explore if USP17 could instead impact upon the lysosome.

To investigate if USP17 impacts upon lysosome trafficking, we transfected HeLa cells with expression vectors for non-targeting (NT), or USP17-specific shRNAs, and knockdown was confirmed by QPCR and Western blotting (Fig EV1A and B). We then assessed the localization of lysosomes via LAMP1 staining using confocal microscopy (Fig 1A and B). In control cells, lysosomes were observed both in the perinuclear region, as well as the periphery of the cell (Fig 1A, left panels). However, USP17 depletion resulted in the lysosomes shifting to a predominantly perinuclear localization (Fig 1A, middle and right panels). Indeed, when we quantified the relative position of the lysosomes to the nucleus (Fig 1C) across multiple cells, there was a significant shift toward the nucleus upon USP17 depletion (Fig 1B, left panel). A similar impact was observed upon the position of LAMP1-positive vesicles in MDA-MB-231 cells transfected as above (Fig EV2A), and again USP17 depletion caused a significant shift of lysosomes toward the nucleus in these cells (Fig 1B, right panel), demonstrating this was not specific to HeLa cells. We also confirmed that the observed change in LAMP1 distribution was not the result of a change in LAMP1 levels, as overexpression of active USP17 did not markedly alter LAMP1 protein levels (Fig EV2B).

We further confirmed this impact upon the lysosome by repeating these experiments in HeLa cells cotransfected with an expression construct for CD63-GFP, another marker of late endosomes (LEs)/lysosomes. Again, we assessed the localization of CD63-GFP via confocal microscopy (Fig 1D). In control cells, the CD63-positive LEs/lysosomes were again observed in the perinuclear region and the periphery of the cell (Fig 1D, left panels). As before, USP17 depletion shifted these LEs/lysosomes to a predominantly perinuclear localization (Fig 1D, middle and right panels). In addition, we cotransfected HeLa cells with an expression construct for LAMP1GFP (Fig EV2C), and examined the relative position of both LAMP1GFP- and CD63-GFP-positive vesicles, and as before they both exhibited a significant shift toward the nucleus upon USP17 depletion (Fig 1E).

To further confirm this impact upon the lysosome, we transfected HeLa cells with either an empty vector, or expression plasmids for USP17 or USP17CS (inactive mutant), and examined endogenous levels of the abundant lysosomal protease cathepsin D (CatD). As had been observed for legumain (Lin et al, 2014; Chen et al, 2019), overexpression of active USP17 results in a drop in intracellular CatD (Fig 1F, bottom panels), again indicating an impact upon the lysosome, rather than just legumain. We further confirmed this drop in intracellular CatD via a fluorescent protease activity assay using a CatD/E-specific substrate. Overexpression of active USP17, but not USP17CS, resulted in a drop in CatD/E activity in lysates taken from these cells (Fig 1G). The addition of the intracellular calcium chelator BAPTA-AM $(30 \mu \mathrm{M}$ for $3 \mathrm{~h}$ ) blunted the impact of USP17 overexpression upon CatD/ E activity, indicating this impact is $\mathrm{Ca}^{2+}$ dependent (Fig EV3A). More interestingly, when we examined extracellular levels of CatD, we found that USP17 overexpression triggered increased secretion (Fig 1F, top panel). This indicated USP17, rather than impacting upon protein stability, was triggering the secretion of CatD, by triggering lysosome exocytosis. In addition, the presence of bands representative of pro- and mature CatD in the supernatant indicated that mature enzyme is present in the supernatant (Fig 1F, top panel).

To further confirm USP17 can facilitate lysosome exocytosis and the secretion of active lysosomal proteases, we undertook a live cell proteolysis assay to examine the impact of USP17 depletion upon cleavage of DQ gelatin, which we have previously shown to be cleaved by cathepsins (Small et al, 2013). The degradation of this substrate in the pericellular environment results in the emission of a bright green fluorescence, which was noticeably decreased in Hela cells transfected with the USP17 shRNAs, when compared to control cells (Fig EV3B). This reinforced the previous observations indicating that USP17 facilitates peripheral trafficking of LEs/lysosomes and its expression can trigger the secretion of lysosomal proteases.

\section{USP17 is required for EGF-driven lysosome exocytosis}

USP17 is induced by multiple stimuli including EGF (Burrows et al, 2004; de la Vega et al, 2011; Jaworski et al, 2014), and we have shown that USP17 expression is required for cell migration triggered by several of these stimuli (de la Vega et al, 2011). Interestingly, EGF triggers peripheral lysosome trafficking, something which is required for EGF-driven cell invasion and motility (Dykes et al, 2017). This suggested USP17 induction by these stimuli could be driving peripheral trafficking of lysosomes to allow cell migration. To confirm this, HeLa cells were transfected with nontargeting (NT), or USP17-specific shRNAs, as indicated, and the cells were either starved or stimulated with EGF, as had been done before (Dykes et al, 2017). We then examined the localization of the lysosome by assessing LAMP1 staining as above (Fig 2A). In the control cells, starvation caused perinuclear accumulation of the lysosomes, and they were redistributed throughout the cell upon EGF treatment (Fig 2A, left panels). However, in the USP17depleted cells, EGF treatment had little or no impact upon lysosome localization (Fig 2A, middle and left panels). This was confirmed by measuring the relative lysosome position across a number of cells in each condition (Fig 2B), which revealed EGF caused a significant peripheral shift in lysosome localization in control cells, but this was severely blunted in USP17-depleted cells (Fig 2B). We further confirmed these observations using HeLa cells cotransfected with CD63-GFP, and again USP17 depletion prevented EGF-driven peripheral trafficking of the CD63-positive vesicles (Fig EV4A and B). 
A

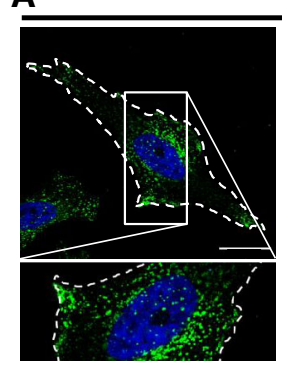

NT shRNA

D

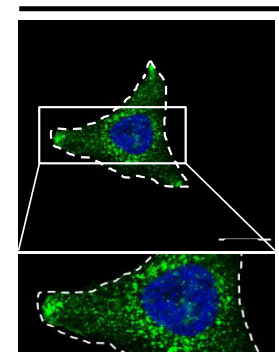

NT shRNA
LAMP1 / DAPI

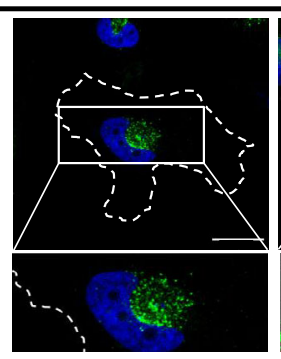

USP17 shRNA1

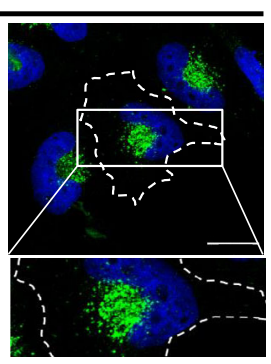

USP17 shRNA2
CD63-GFP / DAPI

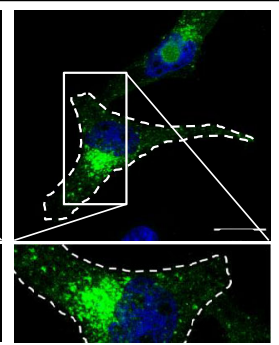

USP17 shRNA1

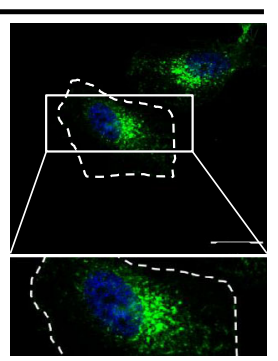

USP17 shRNA2
B

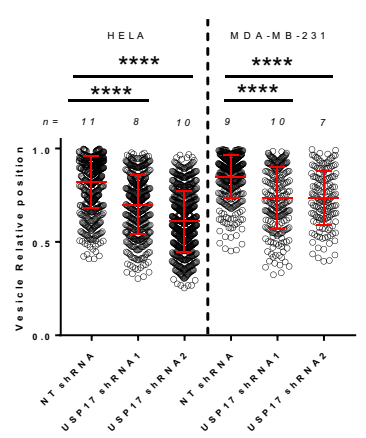

E

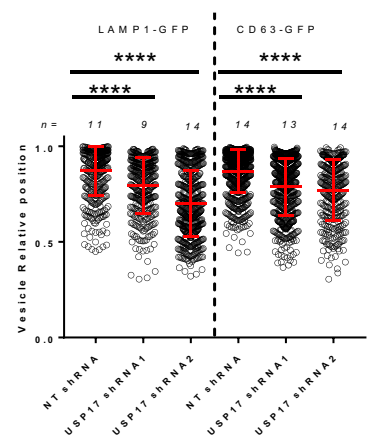

C

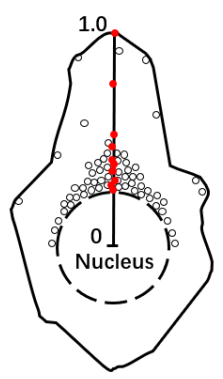

F
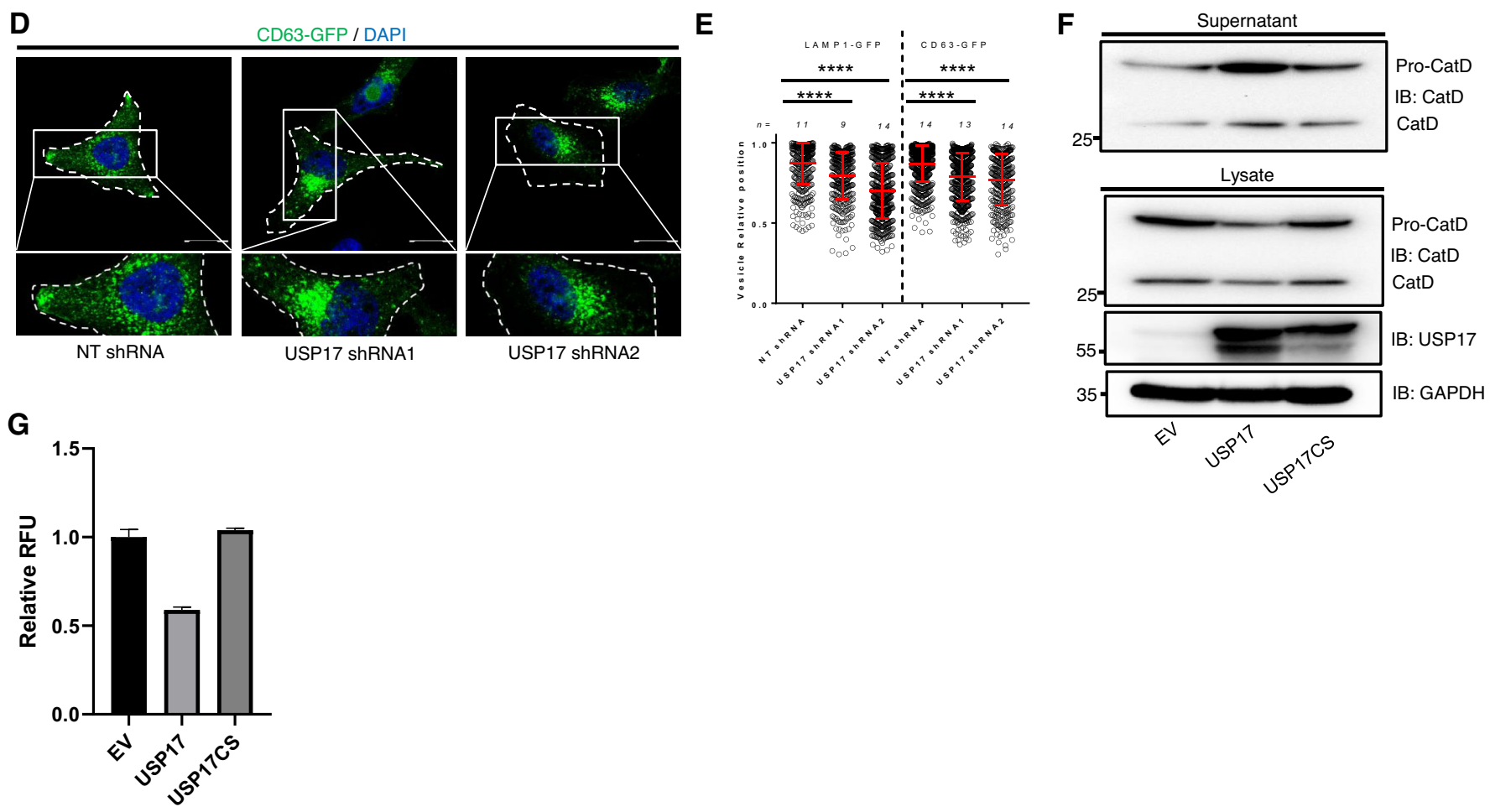

Figure 1. USP17 is necessary for peripheral lysosome trafficking.

A HeLa cells were transfected with constructs for a non-targeting shRNA (NT), USP17 shRNA1, or USP17 shRNA2. Cells were stained using an anti-LAMP1 antibody (green) and the nuclei counterstained with DAPI (blue). Lower panels are enlarged images of indicated area in top panels and the cell membrane is marked by dotted line.

B HeLa and MDA-MB-231 cells were transfected as in A and the distribution of LAMP1-positive vesicles was plotted as vesicle relative position (see C).

C Vesicle relative position is the ratio between vesicle distance from the nucleus border and the sum of the vesicle's distances from the nucleus and cell border. If the vesicle is located within the nucleus, the value is set to 0 . If the value is 1 , then the vesicle is located at the border of cell.

D HeLa cells transfected as in A, in conjunction with a construct coding GFP-tagged CD63. The lower panels are enlarged images of indicated area in top panels and the cell membrane is marked by dotted line.

E HeLa cells transfected as in A, in conjunction with constructs for either GFP-tagged LAMP1 or CD63. The distribution of GFP-positive vesicles plotted as vesicle relative position.

F HeLa cells were transfected with empty vector, or expression constructs for USP17 or USP17CS (inactive mutant). Forty-eight hours post-transfection, lysates and cell growth media were harvested and immunoblotted for GAPDH, USP17, and CatD, as indicated.

G HeLa cells were transfected as in 1F. Forty-eight hours post-transfection, lysates were harvested and $5 \mu \mathrm{g}$ of protein was used in a CatD/E activity assay. Relative fluorescence units (RFU) of triplicate samples at $60 \mathrm{~min}$ compared to empty vector control are plotted. The results plotted are representative of results obtained in three separate experiments.

Data information: In (A, C), scale bars $25 \mu \mathrm{m}$. In (B, D), vesicle position of at least 350 vesicles from a number of cells ( $n$ ) from a series of confocal images across three separate experiments was analyzed using the IMARIS software package and the distribution of vesicles plotted as vesicle relative position (mean value shown as red bar). Error bars represent standard error; and ${ }^{* \star \star *}$ indicates a $P$-value $<0.0001$. One-way ANOVA was used to determine statistically significant differences between groups.

Together, these data indicate USP17 expression is required for LEs/lysosome peripheral trafficking in response to EGF, something which fits with the previous observations that EGF requires lysosome exocytosis to promote cell migration (Dykes et al, 2017), that USP17 is induced by EGF (Jaworski et al, 2014), and USP17 induction is required for cell migration (de la Vega et al, 2011). 
A
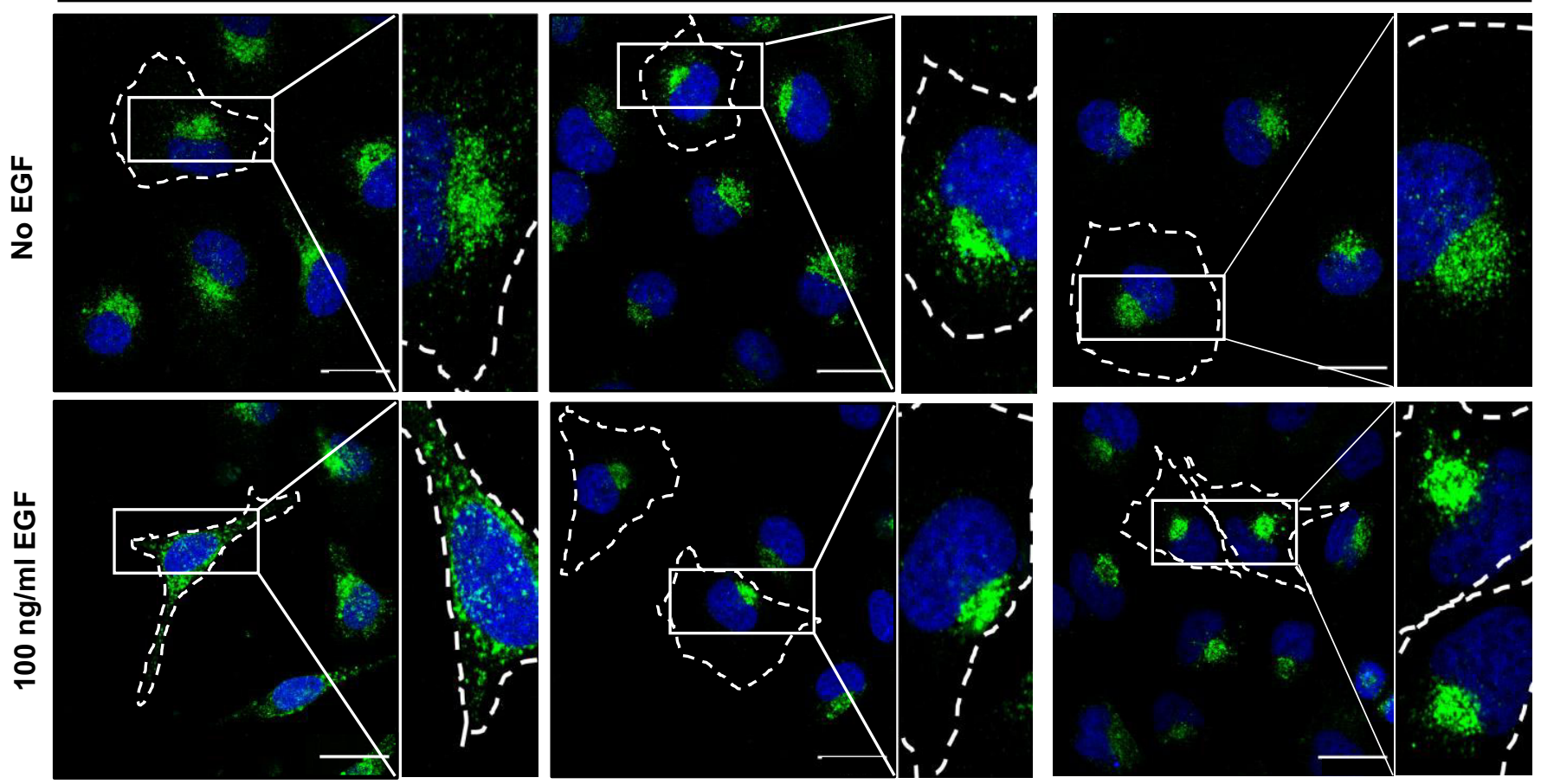

NT shRNA

USP17 shRNA1

USP17 shRNA2

$\mathbf{B}$

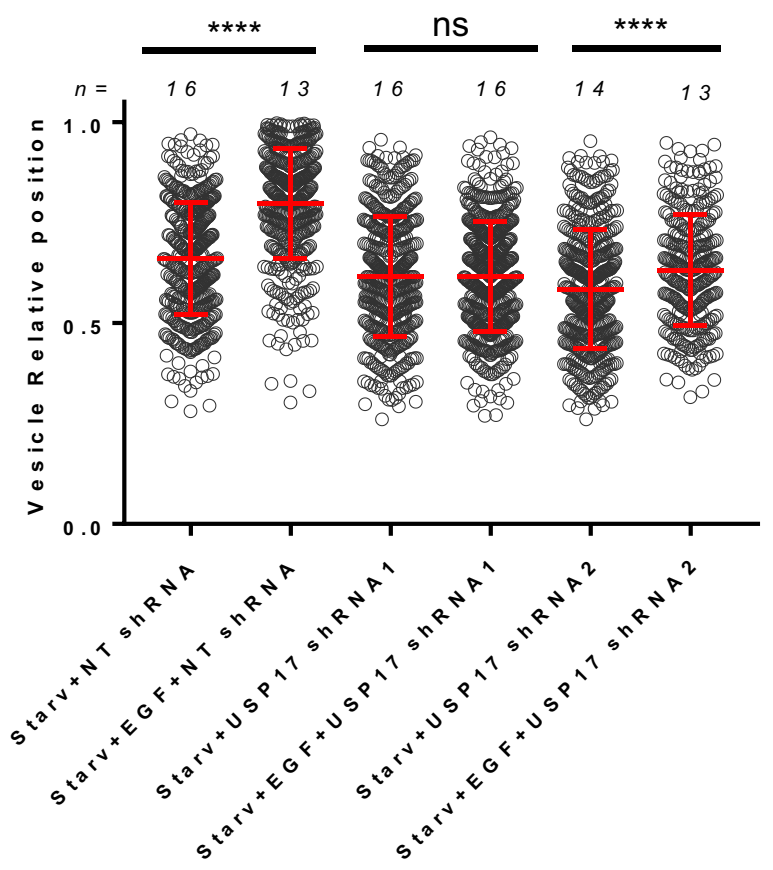

Figure 2. USP17 is necessary for EGF-mediated peripheral lysosome trafficking.

A HeLa cells were transfected with constructs for a non-targeting (NT) shRNA, USP17 shRNA1, or USP17 shRNA2. Forty-eight hours post-transfection, the cells were either serum starved (upper panels), or treated with serum-free medium containing $100 \mu \mathrm{g} / \mathrm{ml}$ EGF (lower panels) for $16 \mathrm{~h}$ prior to staining for LAMP1 (green) and DAPI (blue). Right hand panels are enlarged images of the indicated area in left panels and the cell membrane is marked by dotted line. Scale bars $25 \mu \mathrm{m}$.

B The distribution of at least 300 LAMP1-positive vesicles from a number of cells ( $n$ ) from a series of confocal images across three separate experiments was plotted as vesicle relative position (mean value is red bar). Error bars represent standard error, ns indicates not significant, and ${ }^{* \star \star \star}$ indicates $P$-values $<0.0001$. One-way ANOVA was used to determine statistically significant differences between groups. 


\section{USP17 is required to facilitate plasma membrane repair}

Plasma membrane damage is a frequent event in mammalian cells, especially in cells under mechanical stress, or under attack from invading pathogens which utilize pore-forming toxins, and restoring membrane integrity is essential for cell survival (Andrews \& Perez, 2015). Several mechanisms have been proposed to facilitate plasma membrane repair, one of which is the fusion of lysosomes with the plasma membrane triggered by a damage-induced calcium influx (Reddy et al, 2001). This allows the lysosomal enzyme acid sphingomyelinase to be secreted causing the production of ceramide at the plasma membrane, triggering endocytosis of damaged membrane and restoring plasma membrane integrity (Tam et al, 2010). In addition, secretion of the lysosomal proteases cathepsins $\mathrm{B}, \mathrm{D}$, and $\mathrm{L}$ has also been shown to contribute to the regulation of this process (Castro-Gomes et al, 2016). As we had found USP17 to facilitate peripheral trafficking of LEs/lysosomes, and the secretion of lysosomal proteases, we hypothesized that USP17 would also facilitate proper plasma membrane repair as an important mechanism of plasma membrane repair requires lysosome exocytosis (Reddy et al, 2001). To assess this, we transfected HeLa cells as before with the non-targeting and USP17-specific shRNAs and triggered plasma membrane damage using streptolysin O (SLO), a bacterial protein that forms pores in the plasma membrane and is routinely used to assess plasma membrane repair (Andrews \& Perez, 2015; Encarnação et al, 2016). The cells were initially incubated with SLO in the absence of $\mathrm{Ca}^{2+}$ to prevent repair being initiated, as $\mathrm{Ca}^{2+}$ is required to trigger lysosomal exocytosis and fusion with the plasma membrane. The cells then either remained in the absence of $\mathrm{Ca}^{2+}$ (Fig 3A, middle panels), or were transferred to EBSS buffer containing $\mathrm{Ca}^{2+}$ to allow plasma membrane repair to proceed (Fig 3A, right panels). The cells were then incubated with propidium iodide (PI) and uptake assessed via flow cytometry (Fig 3A). In all cases, some cells in each population exhibited damage prior to SLO treatment due to the observation of PI uptake (Fig 3A, left panels). However, SLO treatment dramatically increased the percentage of cells displaying plasma membrane damage, with over $80 \%$ of the cells assayed showing PI uptake in all populations when these pores were not repaired in the absence of $\mathrm{Ca}^{2+}$ (Fig 3A, middle panels). However, upon the introduction of $\mathrm{Ca}^{2+}$ via EBSS buffer, the control cells reduced PI uptake back to basal levels (Fig 3A, top right panel), indicating the majority of pores triggered by SLO treatment were repaired and that plasma membrane repair was functioning correctly. In contrast, in cells depleted of USP17, the levels of PI uptake remained at two to three times the basal rate even when they were exposed to $\mathrm{Ca}^{2+}$ (Fig 3A, middle and bottom right panels), suggesting plasma membrane repair was not functioning correctly in the absence of USP17. As done previously (Encarnação et al, 2016), we then calculated the percentage plasma membrane repair and observed that this dropped significantly upon USP17 depletion when compared to control cells (Fig 3B). This all indicated that the loss of peripheral lysosomes in the absence of USP17 impairs the ability of these cells to fully repair their plasma membrane when damaged by SLO, and further supported the data indicating that USP17 is required for lysosome exocytosis.

\section{USP17 deubiquitylates p62 and opposes RNF26 to allow lysosome exocytosis}

Lysosome positioning and trafficking is regulated in multiple ways, but the previous localization of USP17 to the ER led us to hypothesize that USP17 regulates lysosome tethering to the ER, something which has previously been demonstrated to be facilitated via a number of mechanisms (Bonifacino \& Neefjes, 2017). One mechanism of lysosomal tethering to the ER is via the E3 ligase RNF26, which ubiquitinates p62 allowing it to act as a bridge between the ER and lysosomes, and tethers them in the perinuclear cloud (Jongsma et al, 2016; Bonifacino \& Neefjes, 2017). Therefore, we examined if USP17 could reverse the impact of RNF26, as this was the only tethering mechanism known to involve ubiquitin. To determine if USP17 opposes the action of RNF26, we again depleted USP17 in combination with RNF26-specific and control siRNAs (Fig EV1C) and then examined LAMP1 localization (Fig 4A). As expected, when RNF26 was depleted using the siRNA, this resulted in the LAMP1-positive lysosomes being distributed more toward the periphery of the cell (Fig 4A, left panels, Fig 4B, lanes 1-2). The addition of the non-targeting control shRNA made little difference to the distribution of the lysosomes in the presence or absence of RNF26 (Fig 4A, middle left panels, Fig 4B, lanes 3, 6). In the control cells, USP17 depletion again caused perinuclear accumulation of the lysosomes (Fig 4A, top right and top middle right panels). However, when RNF26 was also depleted, the lysosomes were distributed more toward the periphery of the cell, and USP17 depletion did not result in perinuclear lysosome accumulation (Fig 4A, bottom middle right and bottom right panels). Indeed, when the relative position of the lysosomes was assessed, USP17 depletion again resulted in a significant shift toward the nucleus in control cells (Fig 4B, lanes 45 ), but in cells which had also been depleted of RNF26, the impact of USP17 depletion was less significant (Fig 4B, lanes 7-8), and where it had an impact it did the opposite, spreading the lysosomes to the periphery (Fig 4B, lane 8).

This indicated USP17 opposed the action of RNF26, and therefore we examined USP17s impact upon p62 ubiquitination. We transfected HeLa cells with the indicated constructs expressing HA-

\section{Figure 3. USP17 is required for plasma membrane repair.}

HeLa cells were transfected with constructs encoding a non-targeting (NT) shRNA, USP17 shRNA1, or USP17 shRNA2, as indicated.

A Forty-eight hours post-transfection, the cells were washed with $\mathrm{Ca}^{2+}$ and $\mathrm{Mg}^{2+}$ free ice-cold $\mathrm{HBSS}$. The different cell populations were split into three aliquots. One aliquot was placed into fresh medium (left panels). SLO was added to other two aliquots and then the toxin-containing medium was removed and the aliquots were either placed into $\mathrm{Ca}^{2+}$ and $\mathrm{Mg}^{2+}$ free HBSS (Middle panels), or EBSS containing $\mathrm{Ca}^{2+}$ and $\mathrm{Mg}^{2+}$ (right panels) prior to staining with propidium iodide and analysis by flow cytometry.

B The percentage plasma membrane repair (PMR) was calculated according to the following formula: 1 - (\% PI-positive cells with Ca ${ }^{2+} / \%$ PI-positive cells without $\left(\mathrm{Ca}^{2+}\right) \times 100$. Three separate experiments were analyzed and plotted. Error bars represent standard error, and * indicates $P$-values $<0.05$. One-way ANOVA was used to determine statistically significant differences between groups. 
A
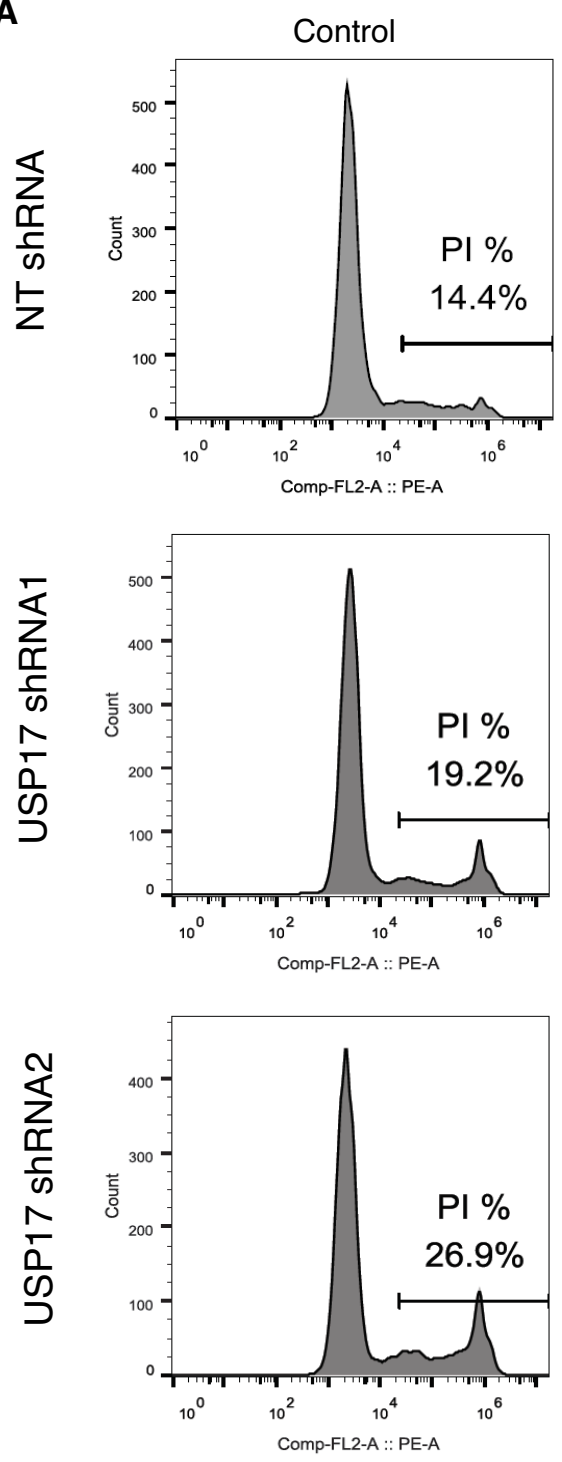

SLO
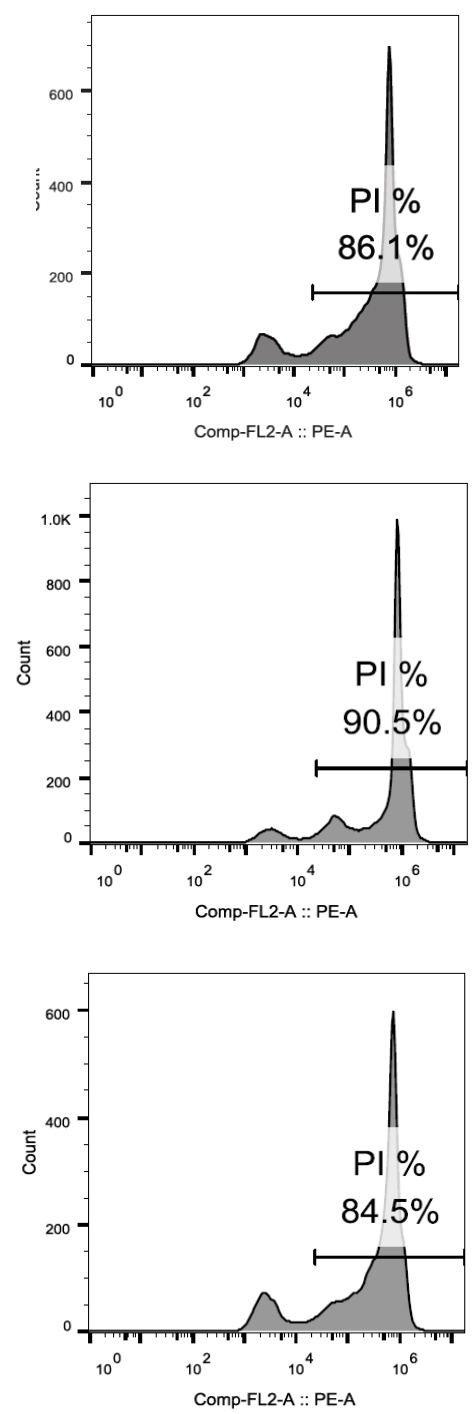

SLO+EBSS
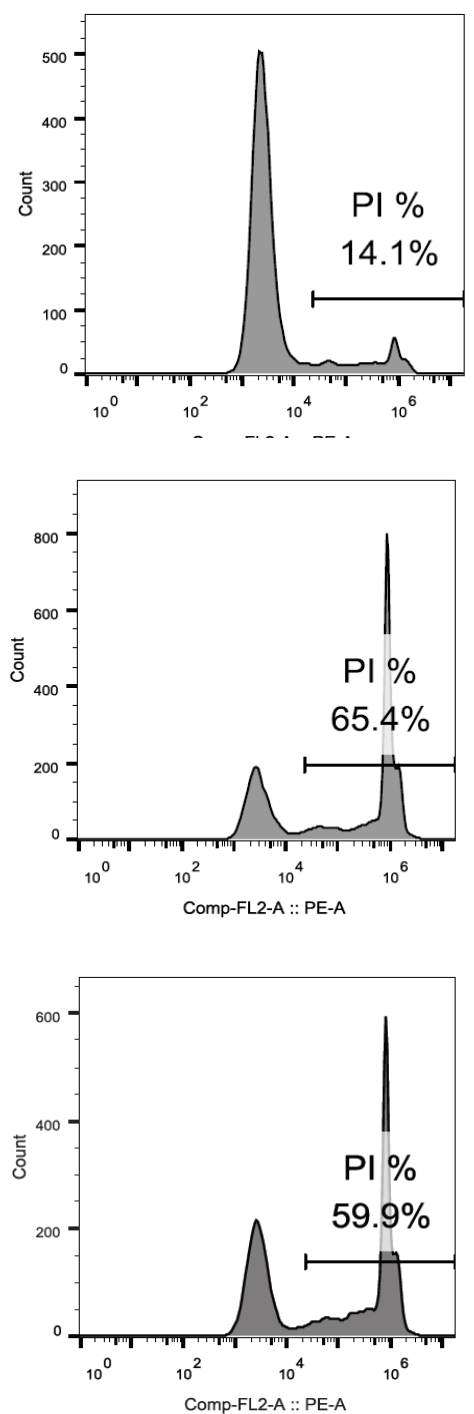

B

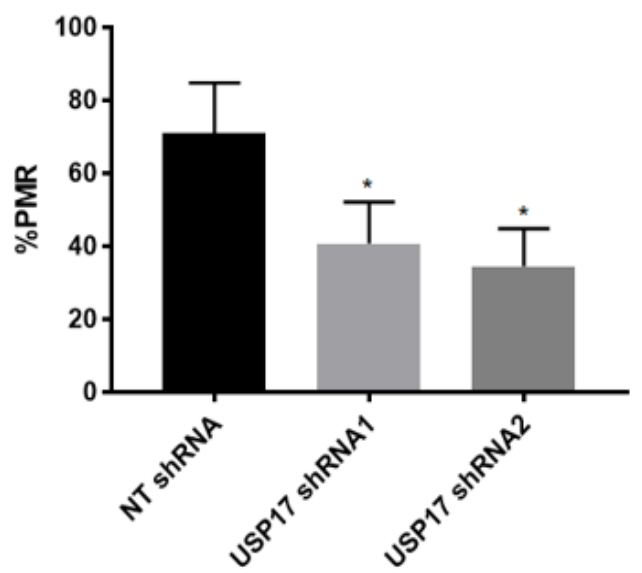

Figure 3. 
A

LAMP1 / DAPI

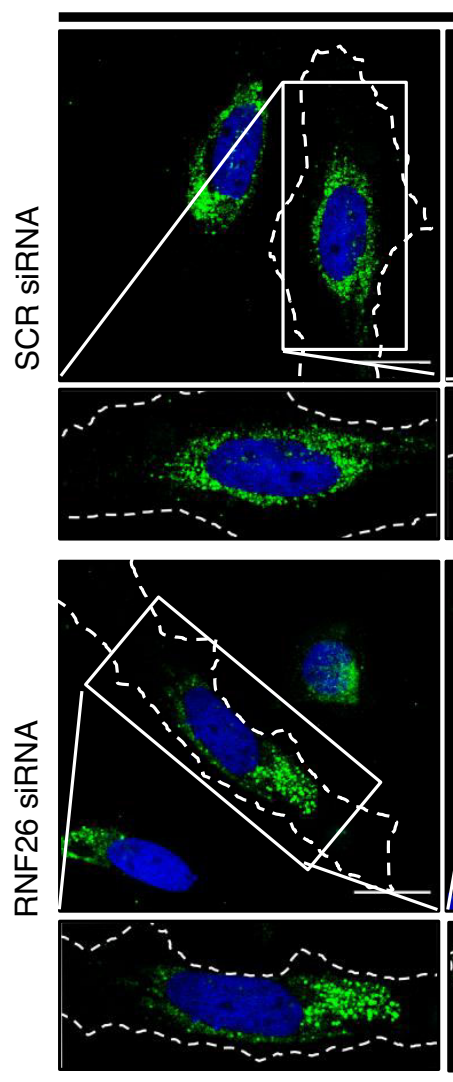

No shRNA
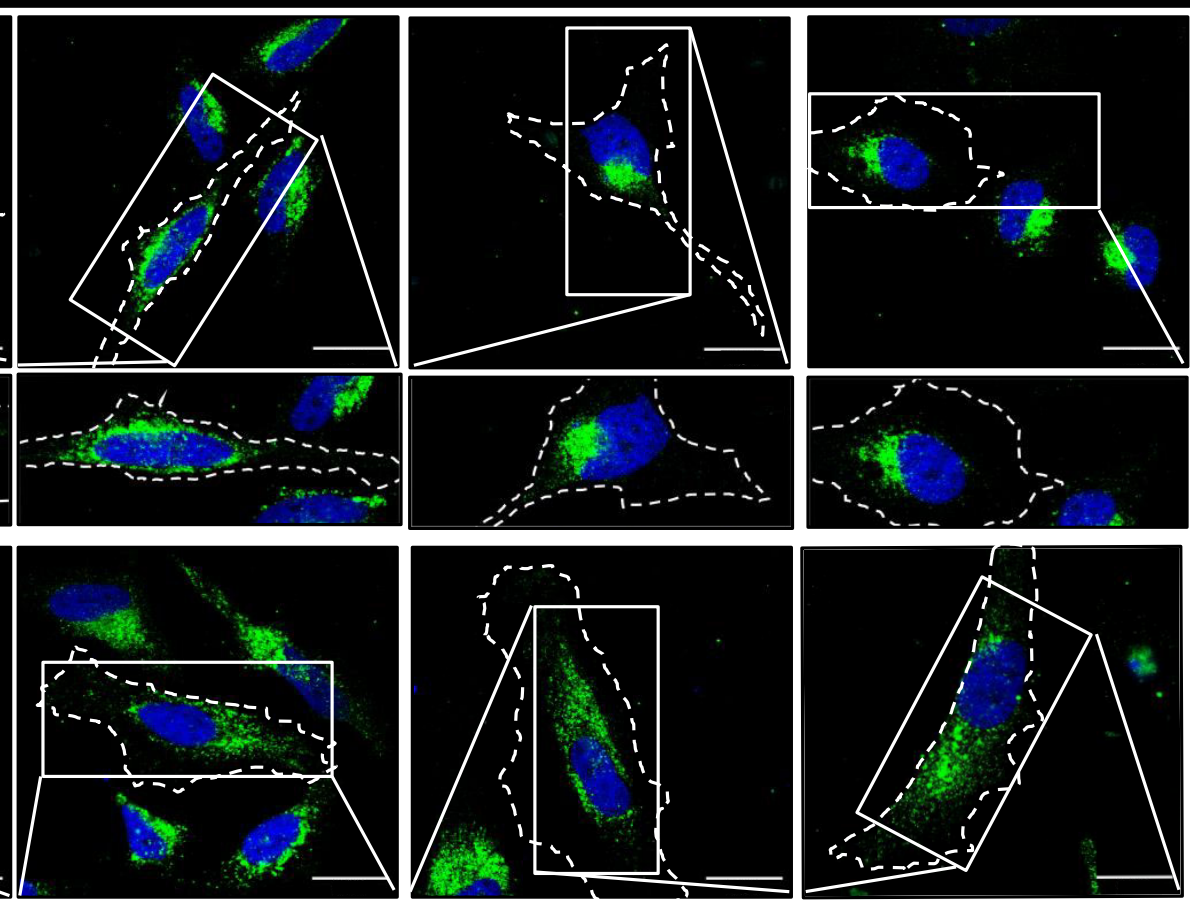

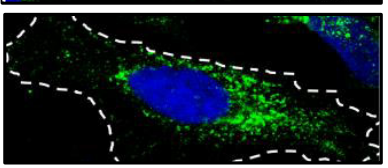

NT shRNA

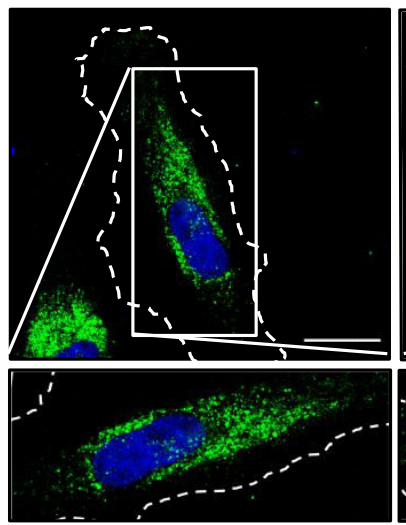

USP17 shRNA1

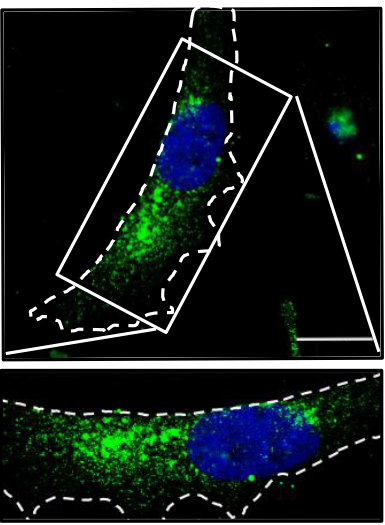

USP17 shRNA2
B

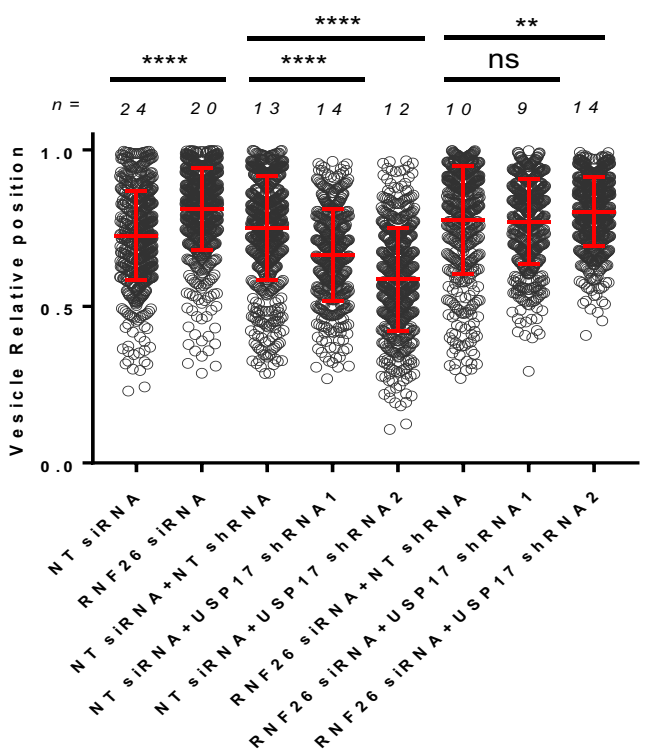

C

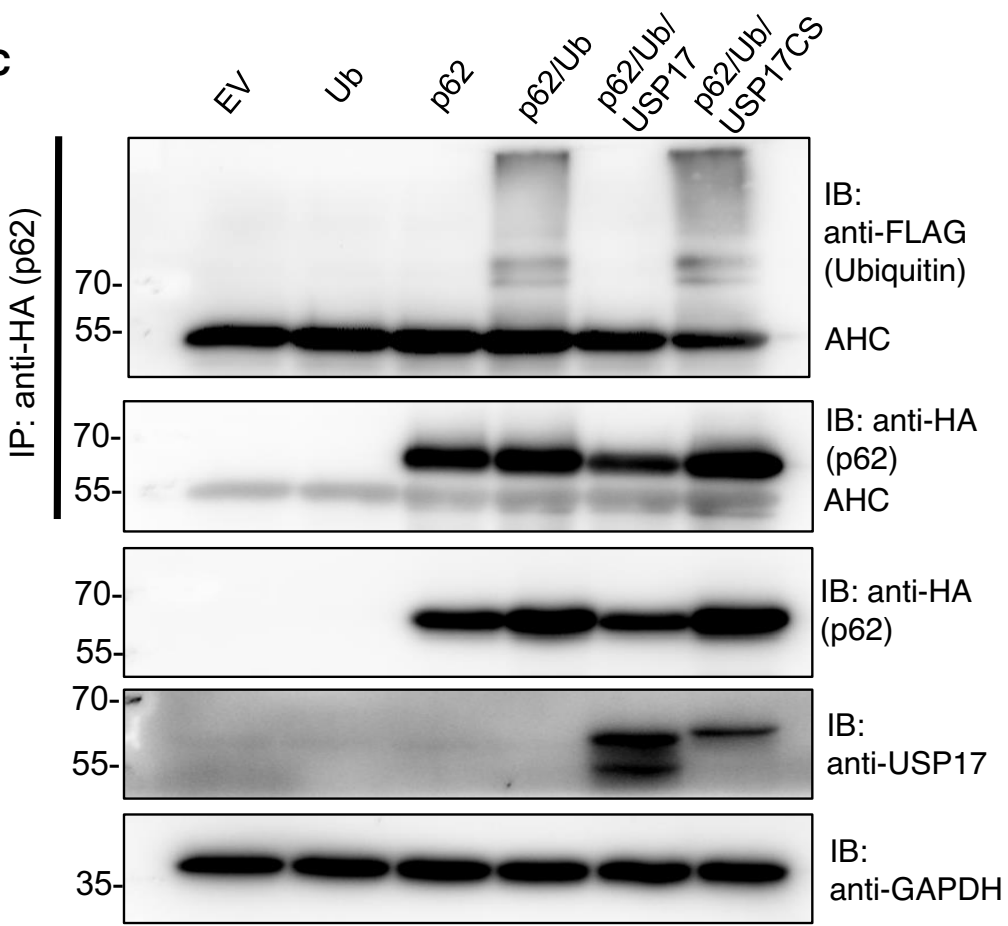

Figure 4. 
Figure 4. USP17 opposes the action of RNF26 and deubiquitylates p62.

A HeLa cells were transfected with negative control siRNA and RNF26 siRNA as well as constructs coding a non-targeting (NT) shRNA, USP17 shRNA1, or USP17 shRNA2, as indicated. Forty-eight hours post-transfection, cells were stained for LAMP-1 (green) and DAPI (blue). Lower panels are enlarged images of indicated area in top panels and the cell membrane is marked by dotted line. Scale bars $25 \mu \mathrm{m}$.

B The distribution of at least 400 LAMP1-positive vesicles from a number of cells ( $n$ ) from a series of confocal images across three separate experiments was plotted as vesicle relative position (mean value is red bar). Error bars represent standard error, ns indicates not significant, ${ }^{* *}$ indicates $P$-values $<0.01$, and ${ }^{* * * *}$ indicates $P$ values $<0.0001$. One-way ANOVA was used to determine statistically significant differences between groups.

C HeLa cells were transfected with expression constructs for HA-tagged p62 and FLAG-tagged ubiquitin as well as empty vector, USP17, or USP17CS. After 48 h, cell lysates were prepared and HA-tagged proteins were pulled down using anti-HA agarose. Pull downs and lysates were immunoblotted with anti-HA, anti-FLAG, and anti-USP17 antibodies, as indicated, to confirm the presence of ubiquitinated p62.

tagged p62, FLAG-tagged ubiquitin, and USP17 or USP17CS, and did pull downs using anti-HA agarose. USP17 expression resulted in a loss of the monoubiquitin from p62, something not observed with the inactive mutant (Fig 4C). This indicates USP17 deubiquinates p62 to release lysosomes from the perinuclear cloud, facilitating their peripheral trafficking, exocytosis, and plasma membrane repair. To further confirm that this impact was due to the monoubiquitination and tethering mechanism, we examined the impact of USP17 overexpression and depletion upon endogenous p62 levels, and observed no marked alteration (Fig EV5A). This indicated USP17 was not regulating p62 turnover, via the proteasome, or autophagy. The was supported further by the observation that treating HeLa cells with the proteasome inhibitor Bortezomib had no obvious impact upon LAMP1 localization (Fig EV5B).

In the original study examining the impact of RNF26 upon lysosome tethering, it was also found to be involved in tethering other endocytic vesicles to the ER, including early endosomes (Jongsma et al, 2016). Therefore, to further confirm that USP17 opposes the action of RNF26, we transfected HeLa cells with non-targeting (NT) and USP17-specific shRNAs, as well as expression constructs for RAB5-GFP or EEA1-GFP, markers of early endosomes. Both of these markers had been shown to be distributed to the cell periphery upon RNF26 depletion, indicating RNF26 tethers early endosomes to the ER (Jongsma et al, 2016). Depletion of USP17 resulted in the clustering of both RAB5 and EEA1-positive endosomes toward the perinuclear region (Fig $5 \mathrm{~A}$ ), and a significant shift was confirmed by measuring the relative position of individual RAB5 and EEA1positive endosomes (Fig 5B). This again indicated USP17 opposes the impact of RNF26 and further reinforces the conclusion that USP17 acts to deubiquitinate p62 and release these vesicles from the perinuclear cloud.

There are a limited number of studies examining RNF26, including two of which indicate RNF26 regulates interferon (IFN) production and STING-dependent innate immune signaling (Qin et al, 2014; Fenech et al, 2020). Interestingly, USP17 has previously been shown to be required for RIG-I/MDA5-dependent IFN production, indicating USP17 may act to counter RNF26 in more than one context (Chen et al, 2010). In addition, RNF26 depletion blocks EGF trafficking to the perinuclear cloud from the cell periphery, as well as EGFR degradation upon EGF engagement, and it redistributes the transferrin receptor (TfR) toward the cell periphery (Jongsma et al, 2016). Two subsequent studies have also indicated RNF26 facilitates the targeting of EGFR to the lysosome for degradation (Cremer et al, 2021; Torrino et al, 2021). We have shown that USP17 is required for clathrin-mediated endocytosis of EGFR and TfR, and that USP17 depletion also blocks EGFR degradation upon EGF engagement, although it blocks its endocytosis, rather than its subsequent targeting to the lysosome (Jaworski et al, 2014). This indicates both RNF26 and USP17 are required to facilitate the trafficking of these receptors, and further reinforces the connection between them.

This molecular mechanism could also potentially explain USP17's role in cancer. As mentioned before, USP17 is overexpressed in a range of tumors when compared to normal tissue (NSCLC, ovarian, breast, colon, esophagus, cervical, and osteosarcoma) (McFarlane et al, 2010, 2013; Pereg et al, 2010; Zhou et al, 2015; Zhang et al, 2016; Song et al, 2017), and high USP17 expression has also been associated with recurrence and metastases in NSCLC (McFarlane et al, 2013). Interestingly, a study which screened for proteins which could drive lung cancer metastases identified the most potent driver of metastases as TMEM106B, which drives lysosome biogenesis and exocytosis, and indicated lysosome exocytosis was required for its ability to drive metastases (Kundu et al, 2018). This could indicate that USP17 overexpression in tumors could drive cell migration by allowing the release of lysosomes to the cell periphery, as seen in response to EGF (Dykes et al, 2017), and this could facilitate metastases.

In the original study which identified RNF26 as the ligase targeting p62, USP15 was also identified as a RNF26 binder and found to deubiquitinate p62 (Jongsma et al, 2016). Silencing USP15 ablated highly mobile peripheral lysosomes marked by lysotracker and resulted in an overall decrease in lysosome mobility, although it did not significantly alter the distribution of CD63-positive LEs/lysosomes (Jongsma et al, 2016). The data presented here indicate USP17 only has an impact in the presence of RNF26, can deubiquitinate $\mathrm{p} 62$, and has a significant impact upon LE/lysosome positioning. This indicates USP17 is able to counteract the ubiquitination of

Figure 5. USP17 is necessary for peripheral trafficking of early endosomes.

A HeLa cells were transfected with constructs for EEA1-GFP and RAB5-GFP in addition to non-targeting scrambled (NT) shRNA, USP17 shRNA1, or USP17 shRNA2. Seventy-two hours post-transfection, the cells were stained with DAPI. Scale bars $25 \mu \mathrm{m}$.

B The distribution of at least 400 EEA1 or RAB5-positive vesicles from a number of cells $(n)$ from a series of confocal images across three separate experiments was plotted as vesicle relative position (mean value is red bar). Error bars represent standard error and $* * *$ indicates a $P$-values $<0.0001$. One-way ANOVA was used to determine statistically significant differences between groups. 
A

EEA1-GFP / DAPI
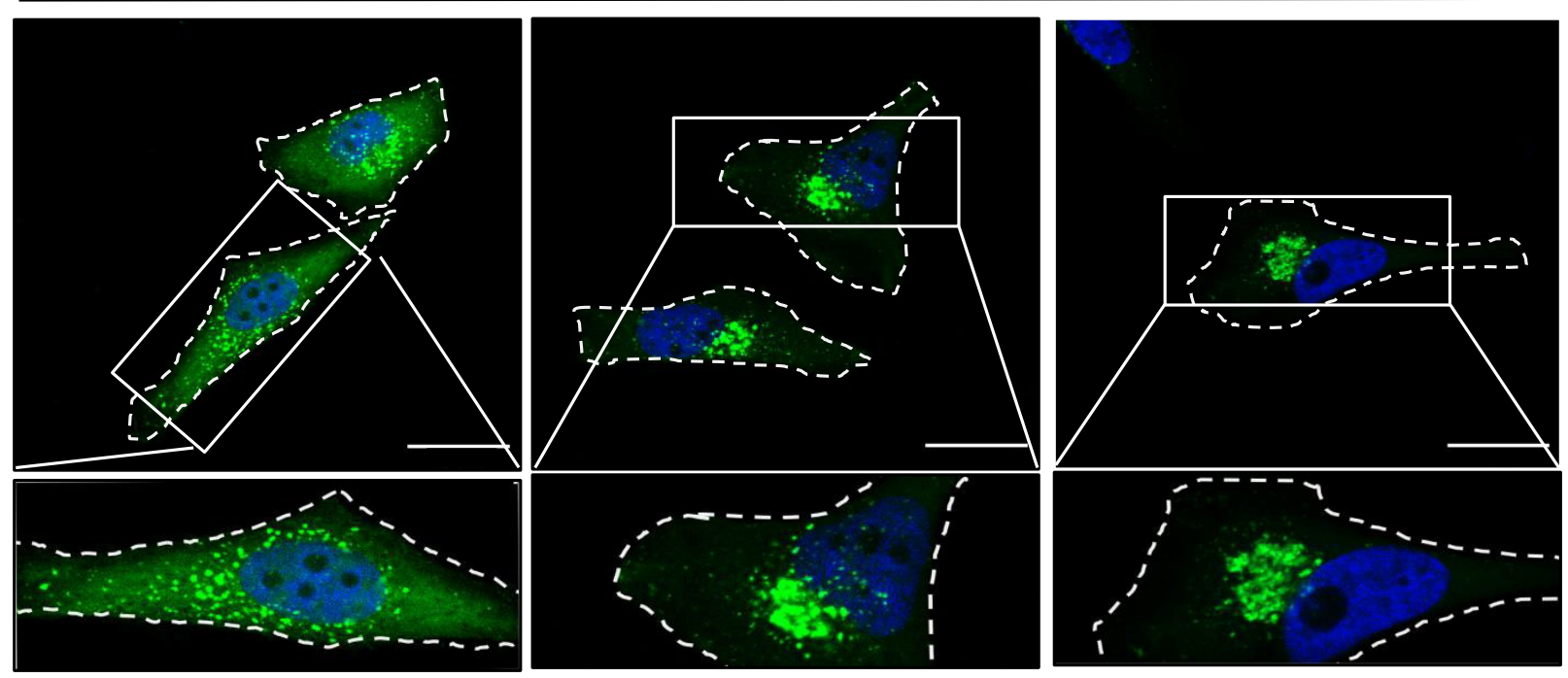

Rab5-GFP / DAPI
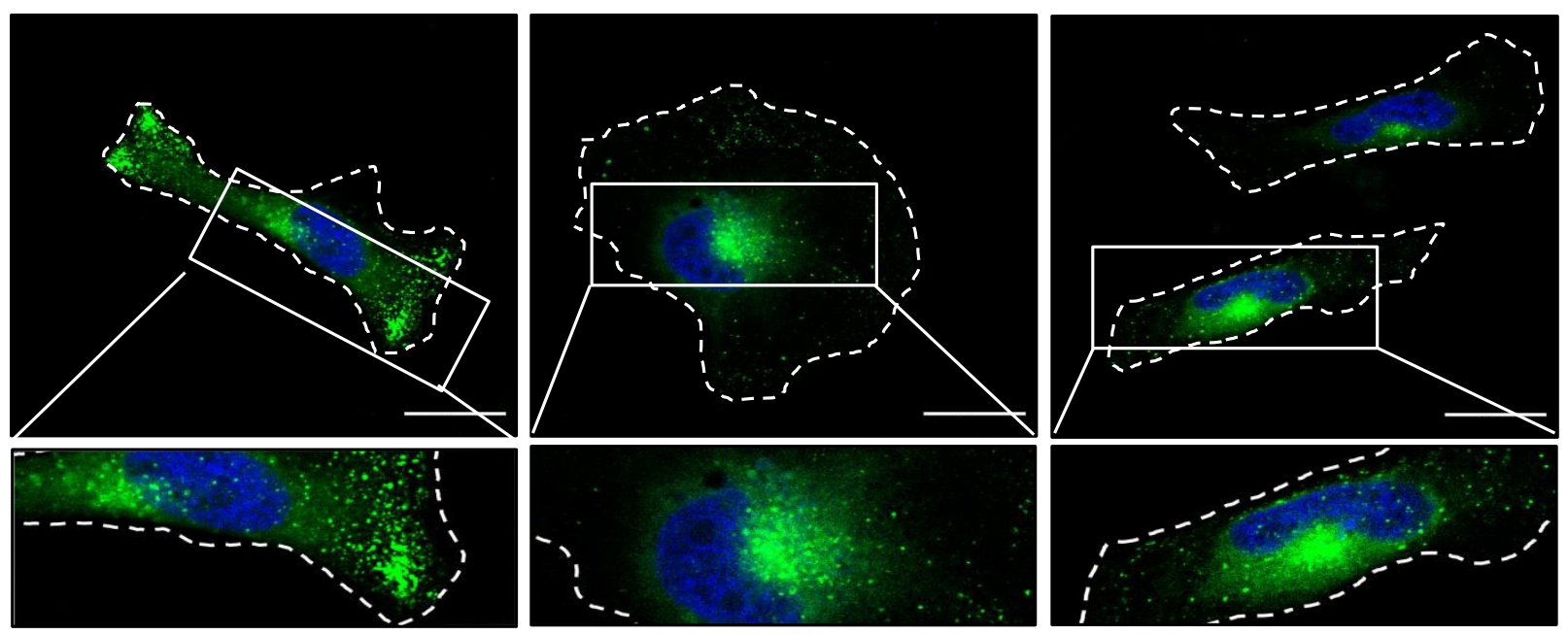

NT shRNA

B

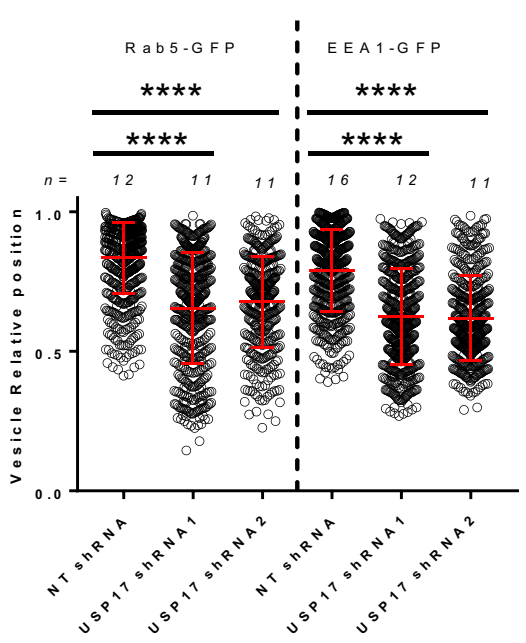

Figure 5.

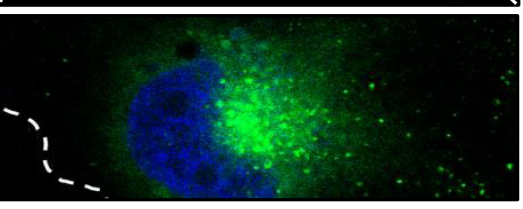

USP17 shRNA1

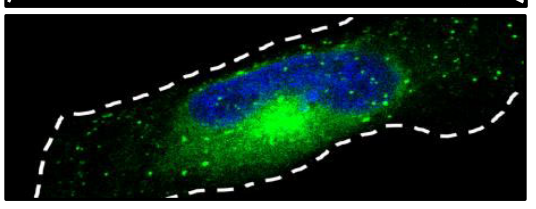

USP17 shRNA2 
p62 by RNF26, and untether LEs/lysosomes from the ER to allow their peripheral trafficking. However, it is possible that both USP15 and USP17 are required to counteract RNF26 ubiquitination of p62, but under different contexts. In particular, USP17, which is induced by multiple extracellular stimuli (Burrows et al, 2004; de la Vega et al, 2011; Jaworski et al, 2014), may be of particular importance in mediating lysosome peripheral trafficking in response to these extracellular signals, as we have shown for EGF. However, further studies are required to further delineate the relationship among RNF26, USP15, and USP17 to determine in which contexts each is important and how each of them contributes to the various different functions which have been attributed to them individually.

\section{Materials and Methods}

\section{Plasmids}

The pDQ-EV, pDQ-USP17, pDQ-USP17CS, USP17 shRNA1 (pSUPERUSP17shRNA), USP17 shRNA2 (pRS-USP17shRNA), and nontargeting shRNA (pRS-scrambled shRNA) were previously described (McFarlane et al, 2010; de la Vega et al, 2011; Jaworski et al, 2014). Constructs for HA-p62/SQSTM1 (Plasmid \#28027), CD63-pEGFP (Plasmid \#62964), and Lamp1-GFP (Plasmid \#34831) were obtained from Addgene. RNF26 and NT siRNA were previously described (Jongsma et al, 2016).

\section{Cell culture and DNA transfections}

HeLa and MDA-MB-231 cells (American Type Culture Collection (ATCC), Manassas, USA) were grown in DMEM supplemented with 10\% FCS, $1 \%$ penicillin $(10,000 \mathrm{U} / \mathrm{ml}) /$ streptomycin $(10,000 \mu \mathrm{g} / \mathrm{ml})$, and $1 \% \mathrm{~L}$ glutamine (200 mM) (Thermo Fisher Scientific, Waltham, USA). Cells lines were grown at $37^{\circ} \mathrm{C}$ in a $5 \% \mathrm{CO}_{2}$ humidified incubator.

Cells were transfected with Lipofectamine 3000 (HeLa; Thermo Fisher Scientific, Waltham, USA) or Xtreme-GENE HP ${ }^{\text {TM }}$ (MDA-MB231; Roche Diagnostics, Indianapolis, USA) transfection reagents according to manufacturer's instructions. Cells were seeded between $0.5 \times 10^{6}$ and $1.0 \times 10^{6}$ cells for cell cycle analysis or protein experiments, or $0.7-2.5 \times 10^{4}$ on four-well glass culture slides (BD Falcon, Bedford, USA) for microscopy experiments. The cells were transfected with $2 \mu \mathrm{g}$ of plasmid DNA for protein experiments and biological assays, or $0.25 \mu \mathrm{g}$ of plasmid DNA for confocal microscopy experiments. For those experiments with EGF stimulation, cells were rested in EBSS (Thermo Fisher Scientific, Waltham, USA) medium without serum and stimulated with $100 \mathrm{ng} / \mathrm{ml}$ recombinant human EGF (Thermo Fisher Scientific, Waltham, USA) for the indicated times as had been done previously (Dykes et al, 2017).

\section{Confocal microscopy}

Cells were seeded at $0.7-2.5 \times 10^{4}$ cells $/ 1.7 \mathrm{~cm}^{2}$ well of glass culture slides (BD Falcon, Bedford, USA). Cells were transfected as previously described. The cells were fixed in $4 \%$ paraformaldehyde (Sigma-Aldrich, Steinheim, Germany), in PBS for $20 \mathrm{~min}$. The cells were then permeabilized in $0.5 \%$ Triton X-100 in PBS for 5 min, washed in PBS, and blocked in blocking solution (1\% BSA, 10\% donkey serum [both from Sigma-Aldrich, Steinheim, Germany] in
PBS) for $1 \mathrm{~h}$ at RT. Transfected proteins and cell organelles were stained with appropriate antibodies or counterstains according to manufacturer's protocol. Antibodies and costains were as follows: mouse anti-LAMP1 (1:1,000; Abcam, Cambridge, UK; Cat no: ab25630) and donkey anti-mouse Alexa Fluor 488 (1:200, Thermo Fisher Scientific-Invitrogen, Waltham, USA; Cat no: A32766). The slides were sealed with a coverslip and Prolong Gold antifade mounting media with DAPI (Thermo Fisher Scientific, Waltham, USA). Fluorescent images were visualized on a Leica TCS SP8 (Leica Microsystems, Milton Keynes, UK) Confocal inverted microscope with an oil immersion objective with $63 \times$ magnification and 1.4 NA (Numerical Aperture). Fluorescent images were captured with a $1024 \times 1024$ frame and $400 \mathrm{~Hz}$ scanning speed. Samples were excited with 405 and $488 \mathrm{~nm}$ line laser and fluorescence emission was collected using spectral HyD detectors. Images were analyzed using Leica LAS X software and images presented in the same figure were captured using standardized settings and exposure times.

\section{Relative vesicle position}

As lysosomes, late endosomes and early endosomes have vesicularlike structure, we can approximate clusters of markers of these vesicles such as LAMP1, CD63, RAB5, and EEA1 with vesicles and use tools to analyze their localization within the cell. Relative vesicle position is the ratio between the vesicle distance from the nucleus border and the sum of the vesicle's distances from the nucleus and cell border. If the vesicle is located within the nucleus, the value is set to 0 . If the value is 1 , then the vesicle is located at the border of the cell. This was calculated using IMARIS 9.3 software (Bitplane AG, Zurich, Switzerland), setting consistent thresholds for cell diameter and vesicles for all images. Thresholds and other parameters were chosen as appropriate based on control samples within each experiment.

\section{DQ-gelatin proteolysis assay}

HeLa cells were transfected as outlined. Transfected cells were trypsinized and placed into a DQ-gelatin/matrigel solution $(25 \mu \mathrm{g} / \mathrm{ml})$ for $24 \mathrm{~h}$ as previously outlined (Small et al, 2013). Cells were then fixed and stained for DAPI (blue), where indicated, before analysis by confocal microscopy.

\section{Plasma membrane repair analysis}

Cell damage was evaluated using propidium iodide (PI) staining, as done before (Encarnação et al, 2016). Cells were washed with HBSS, trypsinized, and $200 \mathrm{ng} / \mathrm{ml}$ streptolysin O (SLO) (Sigma-Aldrich, Steinheim, Germany) was added in $\mathrm{HBSS}\left(\mathrm{Ca}^{2+}\right.$ and $\mathrm{Mg}^{2+}$ free) containing DTT $(10 \mathrm{mM})$. The SLO-containing HBSS was removed and replaced by fresh HBSS $\left(\mathrm{Ca}^{2+}\right.$ free) or EBSS $\left(\mathrm{Ca}^{2+}\right.$ complete) for $10 \mathrm{~min}$ at $37^{\circ} \mathrm{C}$. Cells were then stained using $50 \mu \mathrm{g} / \mathrm{ml}$ propidium iodide (PI) with RNase A $(250 \mu \mathrm{g} / \mathrm{ml})$, incubated at $37^{\circ} \mathrm{C}$ for $30 \mathrm{~min}$, and analyzed by FACS Calibur (BD Biosciences, Franklin Lakes, USA) and FlowJo software.

\section{Cell lysis, pull downs, and immunoblotting}

Cells were lysed in the following buffer: $25 \mathrm{mM}$ Tris-HCl pH 7.6, $150 \mathrm{mM} \mathrm{NaCl}, 1 \% \mathrm{NP}-40,1 \%$ sodium deoxycholate, $0.1 \%$ SDS, 
supplemented with phenylmethylsulfonyl fluoride (1 mM), aprotinin $(1.7 \mu \mathrm{g} / \mathrm{ml})$, and leupeptin $(10 \mu \mathrm{g} / \mathrm{ml})$. Lysates were left on ice for $20 \mathrm{~min}$, centrifuged at $15,000 \mathrm{~g}$ for $10 \mathrm{~min}$ at $4^{\circ} \mathrm{C}$. Protein concentrations were determined and whole cell lysate was diluted into equal volumes and added to Laemlli buffer to a final concentration of $1 \times$ with $5 \% \beta$-mercaptoethanol (Sigma-Aldrich, Steinheim, Germany). Where applicable, equal amounts of lysates were used to pull down HA-tagged proteins using anti-HA agarose before elution and the addition of Laemlli buffer. Subsequently, pull downs and lysate samples were boiled for $5 \mathrm{~min}$ at $95^{\circ} \mathrm{C}$ for protein denaturation. The samples were analyzed by SDS-PAGE and Western blotting on PVDF membrane (Millipore, Waterford, UK). The membranes were then blocked in appropriate blocking agent, either $5 \%$ marvel or $3 \%$ BSA, in $0.1 \%$ Tween-20/PBS for $1 \mathrm{~h}$. After blocking, the membranes were probed with the indicated antibodies for $1 \mathrm{~h}$ at RT or overnight at $4^{\circ} \mathrm{C}$. The following primary antibodies were used: anti-HA $(1: 1,000$, Thermo Fisher Scientific, Waltham, USA; Cat no: 715500), anti-FLAG (1:1,000, Sigma-Aldrich, Steinheim, Germany; Cat no: F3165), anti-GAPDH (1:1000, Bio-techne, Minneapolis, USA; Cat no: AF5718), anti-USP17 (1:2,000, Fusion Antibodies, Belfast, UK; previously used in Burrows et al, 2009; de la Vega et al, 2011; Jaworski et al, 2014), anti-cathepsin D $(1: 1,000$, Abcam, Cambridge, UK; Cat no: ab75852), antitubulin $(1: 1,000$, Abcam, Cambridge, UK; Cat no: ab6160), and anti-LAMP1 (1:1,000, Abcam, Cambridge, UK; Cat no: ab25630). The membrane was incubated with the appropriate secondary antibody: either goat antimouse HRP conjugate, or goat anti-rabbit HRP conjugate (both diluted 1:10,000, Cell Signaling Technology, London, UK; Cat no: anti-mouse (7076S), anti-rabbit (7074S) ) or rabbit anti-rat HRP conjugate (1:40,000, Abcam, Cambridge, UK; Cat no: ab205718). Proteins were detected with a chemiluminescence protocol and were exposed using the ChemiDoc $\mathrm{XRS}^{+}$imaging system (BioRad, Hertfordshire, UK).

\section{RNA extraction and reverse transcription PCR}

RNA was extracted using STAT-60 according to the manufacturer's instructions (Tel-Test Inc., Friendswood, USA). cDNAs were synthesized with the aid of a Transcriptor First Strand cDNA Synthesis kit (Roche Diagnostics, Indianapolis, USA). The Roche LightCycler 96 System was used for real-time PCR analysis employing the cycle threshold $(2-\Delta \Delta \mathrm{CT})$ method. The following primers were used: USP17, 5'-GGCTGCTGGCTTCCATAG-3' (forward) and 5'-CCACAG TGAACATGAGAAATTCA-3' (reverse); RNF26, 5' ${ }^{\prime}$-TCGGCACTCAG AACCTCTTT-3' (forward) and 5'-GCATCACCGACGGAAGGATC-3' (reverse); and GAPDH, 5'-ATGGCAAATTCCATGGCA-3' (forward) and GAPDH $5^{\prime}$-TCTAGACGGCAGGTCAGG-3' (reverse).

\section{Cathepsin D/E activity assay}

HeLa cells were transfected as outlined and lysed using a nondenaturing $50 \mathrm{mM}$ sodium acetate lysis buffer ( $\mathrm{pH}$ 5.5, $0.5 \mathrm{M}$ EDTA, $0.2 \%$ Triton X-100). Lysates were kept on ice for $30 \mathrm{~min}$ (short vortex every $10 \mathrm{~min}$ ), then centrifuged at $15,000 \mathrm{~g}$ for $10 \mathrm{~min}$ at $4^{\circ} \mathrm{C}$. Protein concentration was determined using BCA protein quantification assay. Five microgram of lysis protein in $50 \mu \mathrm{l}$ of sodium acetate buffer was loaded onto a black bottomed 96-well plate, and $50 \mu \mathrm{l}$ of $20 \mu \mathrm{M}$ Cathepsin D/E substrate (Mca-Gly-Lys-Pro-Ile-Leu-
Phe-Phe-Arg-Leu-Lys(Dnp)-D-Arg-NH2) (Enzo Life Sciences, Exeter, UK: BML-P127) was added to each lysate sample. Samples with substrate were subsequently incubated at $37^{\circ} \mathrm{C}$ for $60 \mathrm{~min}$, with fluorescence measured every 2 min (exc: $340 \mathrm{~nm}$; em: $420 \mathrm{~nm}$ ).

\section{Statistical analysis}

Student's $t$-tests and one-way ANOVA were calculated using the GraphPad software (Prism5). P-values were considered statistically significant: $*<0.05, * *<0.01, * * *<0.001$, and $* * * *<0.0001$. $P$ values of $>0.05$ were considered non-significant (ns).

\section{Data availability}

Data sharing is not applicable to this article as no datasets were generated or analyzed during the current study.

Expanded View for this article is available online.

\section{Acknowledgements}

We thank the members of the Burrows, Scott, Burden and Van Schaeybroeck labs, particularly Dr. Peter Smyth, Dr. Francesco Cogo, and Dr. Adam Leach for their technical assistance. This study was partially supported by a grant from the Biotechnology and Biological Sciences Research Council (BBSRC) (Grant number: BB/F013647/1).

\section{Author contributions}

Jia Lin: Data curation; Formal analysis. Aidan P McCann: Data curation; Formal analysis. Naphannop Sereesongsaeng: Data curation; Formal analysis. Jonathan M Burden: Data curation; Methodology. Alhareth A Alsa'd: Data curation; Methodology. Roberta E Burden: Conceptualization; Methodology. Ileana Micu: Conceptualization; Methodology. Richard Williams: Conceptualization; Methodology. Sandra Van Schaeybroeck: Conceptualization. Emma Evergren: Conceptualization. Paul B Mullan: Conceptualization. Jeremy C Simpson: Conceptualization. Christopher J Scott: Conceptualization. James Frederick Burrows: Conceptualization; Data curation; Supervision.

In addition to the CRediT author contributions listed above, the contributions in detail are:

JL, APM, NS, JMB, and AAA carried out the experimental work and participated in manuscript writing. IM assisted with the analysis of the microscopy data. REB, SVS, EE, RW, PM, JCS, and CJS participated in study design and manuscript writing. JFB conceived the study and participated in study design, coordination, and manuscript writing. All authors have read and approved the final manuscript.

\section{Disclosure statement and competing interests}

The authors declare that they have no conflict of interest.

\section{References}

Andrews NW, Perez F (2015) The plasma membrane repair shop: fixing the damage. Semin Cell Deu Biol 45: 10-17

Bonam SR, Wang F, Muller S (2019) Lysosomes as a therapeutic target. Nat Reu Drug Discou 18: 923-948

Bonifacino JS, Neefjes J (2017) Moving and positioning the endolysosomal system. Curr Opin Cell Biol 47: 1-8 
Burrows JF, Kelvin AA, McFarlane C, Burden RE, McGrattan MJ, De la Vega M, Govender U, Quinn DJ, Dib K, Gadina M et al (2009) USP17 regulates Ras activation and cell proliferation by blocking RCE1 activity. J Biol Chem 284: 9587-9595

Burrows JF, McGrattan MJ, Rascle A, Humbert M, Baek KH, Johnston JA (2004) DUB-3, a cytokine-inducible deubiquitinating enzyme that blocks proliferation. J Biol Chem 279: 13993-14000

Castro-Gomes T, Corrotte M, Tam C, Andrews NW (2016) Plasma membrane repair is regulated extracellularly by proteases released from lysosomes. PLoS One 11: e0152583

Cellerino A, Ori A (2017) What have we learned on aging from omics studies? Semin Cell Deu Biol 70: 177-189

Chen R, Zhang L, Zhong B, Tan B, Liu Y, Shu HB (2010) The ubiquitin-specific protease 17 is involved in virus-triggered type I IFN signaling. Cell Res 20: $802-811$

Chen X, Wang C, Liao K, Zhou S, Cao L, Chen J, Xu C, Lin Y (2019) USP17 suppresses tumorigenesis and tumor growth through deubiquitinating AEP. Int J Biol Sci 15: $738-748$

Cremer T, Jongsma MLM, Trulsson F, Vertegaal ACO, Neefjes J, Berlin I (2021) The ER-embedded UBE2]1/RNF26 ubiquitylation complex exerts spatiotemporal control over the endolysosomal pathway. Cell Rep 34: 108659

de la Vega M, Kelvin AA, Dunican DJ, McFarlane C, Burrows JF, Jaworski J, Stevenson NJ, Dib K, Rappoport JZ, Scott CJ et al (2011) The deubiquitinating enzyme USP17 is essential for GTPase subcellular localization and cell motility. Nat Commun 2: 259

Dykes SS, Steffan JJ, Cardelli JA (2017) Lysosome trafficking is necessary for EGF-driven invasion and is regulated by $\mathrm{p} 38 \mathrm{MAPK}$ and $\mathrm{Na}+/ \mathrm{H}+$ exchangers. BMC Cancer 17: 672

Encarnação M, Espada L, Escrevente C, Mateus D, Ramalho J, Michelet X, Santarino I, Hsu VW, Brenner MB, Barral DC et al (2016) A Rab3adependent complex essential for lysosome positioning and plasma membrane repair. J Cell Biol 213: 631-640

Fenech EJ, Lari F, Charles PD, Fischer R, Laétitia-Thézénas M, Bagola K, Paton AW, Paton JC, Gyrd-Hansen M, Kessler BM et al (2020) Interaction mapping of endoplasmic reticulum ubiquitin ligases identifies modulators of innate immune signalling. Elife 9: e57306

Jaworski J, de la Vega M, Fletcher SJ, McFarlane C, Greene MK, Smyth AW, Van Schaeybroeck S, Johnston JA, Scott CJ, Rappoport JZ et al (2014) USP17 is required for clathrin mediated endocytosis of epidermal growth factor receptor. Oncotarget 5: 6964-6975

Jongsma M, Berlin I, Wijdeven R, Janssen L, Janssen C, Garstka M, Janssen H, Mensink M, van Veelen P, Spaapen R et al (2016) An ER-associated pathway defines endosomal architecture for controlled cargo transport. Cell 166: 152-166

Kundu ST, Grzeskowiak CL, Fradette JJ, Gibson LA, Rodriguez LB, Creighton CJ, Scott KL, Gibbons DL (2018) TMEM106B drives lung cancer metastasis by inducing TFEB-dependent lysosome synthesis and secretion of cathepsins. Nat Commun 9: 2731

Lin Y, Qiu Y, Xu C, Liu Q, Peng B, Kaufmann GF, Chen XI, Lan B, Wei C, Lu D et al (2014) Functional role of asparaginyl endopeptidase ubiquitination by TRAF6 in tumor invasion and metastasis. J Natl Cancer Inst 106: dju012
McFarlane C, Kelvin AA, De La Vega M, Govender U, Scott CJ, Burrows JF, Johnston JA (2010) The deubiquitinating enzyme USP17 is highly expressed in tumor biopsies, is cell cycle regulated, and is required for G1S progression. Cancer Res 70: 3329-3339

McFarlane C, McFarlane S, Paul I, Arthur K, Scheaff M, Kerr K, Stevenson M, Fennell DA, Johnston JA (2013) The deubiquitinating enzyme USP17 is associated with non-small cell lung cancer (NSCLC) recurrence and metastasis. Oncotarget 4: 1836-1843

Pereg Y, Liu BY, O’Rourke KM, Sagolla M, Dey A, Komuves L, French DM, Dixit VM (2010) Ubiquitin hydrolase Dub3 promotes oncogenic transformation by stabilizing Cdc25A. Nat Cell Biol 12: 400-406

Qin Y, Zhou MT, Hu MM, Hu YH, Zhang J, Guo L, Zhong B, Shu HB (2014) RNF26 temporally regulates virus-triggered type I interferon induction by two distinct mechanisms. PLoS Pathog 10: e1004358

Reddy A, Caler EV, Andrews NW (2001) Plasma membrane repair is mediated by $\mathrm{Ca}(2+)$-regulated exocytosis of lysosomes. Cell 106: 157-169

Samie MA, Xu H (2014) Lysosomal exocytosis and lipid storage disorders. J Lipid Res 55: 995-1009

Small DM, Burden RE, Jaworski J, Hegarty SM, Spence S, Burrows JF, McFarlane C, Kissenpfennig A, McCarthy HO, Johnston JA et al (2013) Cathepsin S from both tumor and tumor-associated cells promote cancer growth and neovascularization. Int J Cancer 133: 2102-2112

Song C, Liu W, Li J (2017) USP17 is upregulated in osteosarcoma and promotes cell proliferation, metastasis, and epithelial-mesenchymal transition through stabilizing SMAD4. Tumour Biol 39: 1010428317717138

Tam C, Idone V, Devlin C, Fernandes MC, Flannery A, He X, Schuchman E, Tabas I, Andrews NW (2010) Exocytosis of acid sphingomyelinase by wounded cells promotes endocytosis and plasma membrane repair. J Cell Biol 189: 1027-1038

Torrino S, Tiroille V, Dolfi B, Dufies M, Hinault C, Bonesso L, Dagnino S, Uhler J, Irondelle M, Gay A-S et al (2021) UBTD1 regulates ceramide balance and endolysosomal positioning to coordinate EGFR signaling. Elife 10: e68348

Zhang S, Yuan J, Zheng R (2016) Suppression of ubiquitin-specific peptidase 17 (USP17) inhibits tumorigenesis and invasion in non-small cell lung cancer cells. Oncol Res 24: 263-269

Zhou B, Shu B, Xi T, Su N, Liu J (2015) Dub3 expression correlates with tumor progression and poor prognosis in human epithelial ovarian cancer. Biomed Pharmacother 70: 84-89

Zhu Y, Lambert K, Corless C, Copeland NG, Gilbert DJ, Jenkins NA, D'Andrea AD (1997) DUB-2 is a member of a novel family of cytokine-inducible deubiquitinating enzymes. J Biol Chem 272: $51-57$

Zhu Y, Pless M, Inhorn R, Mathey-Prevot B, D'Andrea AD (1996) The murine DUB-1 gene is specifically induced by the betac subunit of interleukin-3 receptor. Mol Cell Biol 16: $4808-4817$

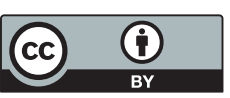

License: This is an open access article under the terms of the Creative Commons Attribution License, which permits use, distribution and reproduction in any medium, provided the original work is properly cited. 